\title{
Gap Equations and Electroweak Symmetry Breaking
}

\author{
G. Cynolter and E. Lendvai \\ Theoretical Physics Research Group of Hungarian Academy of Sciences, \\ Eötvös University, Budapest, 1117 Pázmány Péter sétány 1/A, Hungary
}

\begin{abstract}
Recently a new dynamical symmetry breaking model of electroweak interactions was proposed based on interacting fermions. Two fermions of different $S U_{L}(2)$ representations form a symmetry breaking condensate and generate the lepton and quark masses. The weak gauge bosons get their usual standard model masses from a gauge invariant Lagrangian of a composite doublet scalar field. The new fermion fields become massive by condensation. In this note the gap equations are given in the linearized (mean field) approximation and the conditions for symmetry breaking and mass generation are presented. Perturbative unitarity constrains the selfcouplings and the masses of the new fermions, a raw spectrum is given.
\end{abstract}

With the advent of the LHC the problem of electroweak symmetry breaking becomes more and more important. The LHC experiments are expected to shed light on the dynamics of the symmetry breaking. As there is no direct evidence for elementary scalar (Higgs) particles, alternative, dynamical mechanisms have also been investigated, like technicolour, top condensate or topcolour models [1, 2, 3]. These models were reborn in extra dimensional scenarios like higgsless models 4. Recently a new dynamical symmetry breaking model was proposed based on new fermions of different representations of the weak gauge group [5], in the model four-fermion interactions generate the (symmetry breaking) condensates. The condensates generate masses for the new fermions and an auxiliary composite scalar field is responsible for the weak gauge boson masses. The model is non-renormalizable and regulated by a four dimensional cutoff, we present the model after the introduction in more detail. In this work we extend the model to include more general condensates and investigate the nonconventional structure of the gap equations in the modified model. We were able to analyse the coupled gap equations and give the condition of finding a symmetry breaking solution and fulfilling the constraints of perturbative unitarity. The possible masses for various coupling constants are presented and it is shown that the model can generate fermions with a mass of few hundred $\mathrm{GeV}$ for cutoffs in the TeV range.

To start with we summarize the fermion condensate model of electroweak interactions [5]. In the model to be outlined the Higgs sector is replaced with new 
fermions with non-renormalizable four-fermion interactions. Under $S U_{L}(2) \times$ $U_{Y}(1)$ the new fermions are a neutral singlet $\Psi_{S}$, and a weak doublet $\Psi_{D}=$ $\left(\begin{array}{c}\Psi_{D}^{+} \\ \Psi_{D}^{0}\end{array}\right)$ with hypercharge 1. $\Psi_{D}^{+}\left(\Psi_{D}^{0}\right)$ is a positively charged (neutral) field. The new fermions have effective four-fermion interactions, valid up to some physical cutoff, the ultraviolet completion of the model is not specified.

The new Lagrangian with gauge invariant kinetic terms and invariant 4fermion interactions of the new fermions is $L_{\Psi}$,

$$
\begin{aligned}
L_{\Psi}= & i \bar{\Psi}_{D} D_{\mu} \gamma^{\mu} \Psi_{D}+i \bar{\Psi}_{S} \partial_{\mu} \gamma^{\mu} \Psi_{S}-m_{0 D} \bar{\Psi}_{D} \Psi_{D}-m_{0 S} \bar{\Psi}_{S} \Psi_{S}+ \\
& +\lambda_{1}\left(\bar{\Psi}_{D} \Psi_{D}\right)^{2}+\lambda_{2}\left(\bar{\Psi}_{S} \Psi_{S}\right)^{2}+2 \lambda_{3}\left(\bar{\Psi}_{D} \Psi_{D}\right)\left(\bar{\Psi}_{S} \Psi_{S}\right)
\end{aligned}
$$

$m_{0 D}, m_{0 S}$ are bare masses and $D_{\mu}$ is the covariant derivative

$$
D_{\mu}=\partial_{\mu}-i \frac{g}{2} \underline{\tau} \underline{A}_{\mu}-i \frac{g^{\prime}}{2} B_{\mu},
$$

where $\underline{A}_{\mu}, B_{\mu}$ and $g, g^{\prime}$ are the usual weak gauge boson fields and couplings, respectively. Additional four-fermion couplings are possible but those will not basically change the symmetry breaking and mass generation. We will show in what follows that for couplings $\lambda_{i}$ exceeding the critical value the four-fermion interactions of (11) generate condensates

$$
\begin{aligned}
\left\langle\bar{\Psi}_{D \alpha}^{0} \Psi_{D \beta}^{0}\right\rangle_{0} & =a_{1} \delta_{\alpha \beta}, \\
\left\langle\bar{\Psi}_{D \alpha}^{+} \Psi_{D \beta}^{+}\right\rangle_{0} & =a_{+} \delta_{\alpha \beta}, \\
\left\langle\bar{\Psi}_{S \alpha} \Psi_{S \beta}\right\rangle_{0} & =a_{2} \delta_{\alpha \beta}, \\
\left\langle\bar{\Psi}_{S} \Psi_{D}\right\rangle_{0}=\left\langle\left(\begin{array}{c}
\bar{\Psi}_{S} \Psi_{D}^{+} \\
\bar{\Psi}_{S} \Psi_{D}^{0}
\end{array}\right)\right\rangle_{0} \neq 0 & \neq 0
\end{aligned}
$$

The formation of the charged condensate (4) is more general then in [5], but it does not change the original arguments about symmetry breaking. The nondiagonal condensate in (6) spontaneously breaks $S U_{L}(2) \times U_{Y}(1)$ to $U_{e m}(1)$ of electromagnetism. With the gauge transformations of $\Psi_{D}$ the condensate (6) can always be transformed into a real lower component,

$$
\left\langle\bar{\Psi}_{S \alpha} \Psi_{D \beta}^{0}\right\rangle_{0}=a_{3} \delta_{\alpha \beta}, \quad\left\langle\bar{\Psi}_{S \alpha} \Psi_{D \beta}^{+}\right\rangle_{0}=0,
$$

where $a_{3}$ is real. The composite operator $\bar{\Psi}_{S} \Psi_{D}$ resembles the standard scalar doublet. Assuming invariant four-fermion interactions for the new and known fermions,

$$
L_{f}=g_{f}\left(\bar{\Psi}_{L}^{f} \Psi_{R}^{f}\right)\left(\bar{\Psi}_{S} \Psi_{D}\right)+g_{f}\left(\bar{\Psi}_{R}^{f} \Psi_{L}^{f}\right)\left(\bar{\Psi}_{D} \Psi_{S}\right)
$$

the condensate (7) generates masses to the standard femions. In the linearized, mean field approximation the electron mass, for example, is

$$
m_{e}=-4 g_{e} a_{3} .
$$

Up type quark masses can be generated via the charge conjugate field $\widetilde{\Psi}_{D}=$ $i \tau_{2}\left(\Psi_{D}\right)^{\dagger}$. 
The masses of the weak gauge bosons arise from the effective interactions of the auxiliary composite $Y=1$ scalar doublet,

$$
\Phi=\left(\begin{array}{c}
\Phi^{+} \\
\Phi^{0}
\end{array}\right)=\bar{\Psi}_{S} \Psi_{D}
$$

$\Phi$ develops a gauge invariant kinetic term in the low energy effective description

$$
L_{H}=h\left(D_{\mu} \Phi\right)^{\dagger}\left(D^{\mu} \Phi\right),
$$

where $D_{\mu}$ is the usual covariant derivative (2).

The coupling constant $h$ sets the dimension of $L_{H},[h]=-4$ in mass dimension, we assume $h>0$. (11) is a non-renormalizable Lagrangian and it provides the weak gauge boson masses and some of the interactions of the new fermions with the standard gauge bosons.

The terms with $\Phi^{0}$ in $L_{H}$ can be written as

$$
\begin{aligned}
h^{-1} L_{H}= & \frac{g^{2}}{2} W_{\mu}^{-} W^{+\mu} \Phi^{0 \dagger} \Phi^{0}+\frac{g^{2}}{4 \cdot \cos ^{2} \theta_{W}} Z_{\mu} Z^{\mu} \Phi^{0 \dagger} \Phi^{0}+ \\
& +\left[\partial^{\mu} \Phi^{0 \dagger} \partial_{\mu} \Phi^{0}-\frac{i}{2} \frac{g}{\cos \theta_{W}}\left(\partial^{\mu} \Phi^{0 \dagger}\right) \Phi^{0} Z_{\mu}+\frac{i}{2} \frac{g}{\cos \theta_{W}} \Phi^{0 \dagger} Z_{\mu}\left(\partial^{\mu} \Phi^{0}\right)\right]
\end{aligned}
$$

in terms of the usual vector boson fields.

In the linearized approximation in (12) we put

$$
h \Phi^{0 \dagger} \Phi^{0} \rightarrow h\left\langle\Phi^{0 \dagger} \Phi^{0}\right\rangle_{0}=h\left(16 a_{3}^{2}-4 a_{1} a_{2}\right)=\frac{v^{2}}{2},
$$

leading to the standard masses

$$
m_{W}=\frac{g v}{2}, \quad m_{Z}=\frac{g v}{2 \cos \theta_{W}} .
$$

$v^{2}$ is, as usual, $\left(\sqrt{2} G_{F}\right)^{-1} ; v=254 \mathrm{GeV}$. The tree masses naturally fulfill the important relation $\rho_{\text {tree }}=1$.

Once the condensates (36) are formed, dynamical mass terms are generated in the Lagrangian (11) beside the bare mass terms.

$$
L_{\psi} \rightarrow L_{\Psi}^{\operatorname{lin}}=-m_{+} \overline{\Psi_{D}^{+}} \Psi_{D}^{+}-m_{1} \overline{\Psi_{D}^{0}} \Psi_{D}^{0}-m_{2} \bar{\Psi}_{S} \Psi_{S}-m_{3}\left(\overline{\Psi^{0}}{ }_{D} \Psi_{S}+\bar{\Psi}_{S} \Psi_{D}^{0}\right)
$$

with

$$
\begin{aligned}
m_{+} & =m_{0 D}-6 \lambda_{1} a_{+}-8\left(\lambda_{1} a_{1}+\lambda_{3} a_{2}\right)=m_{1}+2 \lambda_{1}\left(a_{+}-a_{1}\right) \\
m_{1} & =m_{0 D}-6 \lambda_{1} a_{1}-8\left(\lambda_{1} a_{+}+\lambda_{3} a_{2}\right), \\
m_{2} & =m_{0 S}-6 \lambda_{2} a_{2}-8 \lambda_{3}\left(a_{1}+a_{+}\right), \\
m_{3} & =2 \lambda_{3} a_{3} .
\end{aligned}
$$

If $m_{3}=0\left(\lambda_{3}=0\right.$ or $\left.a_{3}=0\right)$ then (15) is diagonal, the original gauge eigenstates are the physical fields, the electroweak symmetry is not broken, $\lambda_{3} a_{3}$, the nondiagonal condensate triggers the mixing and symmetry breaking. If $m_{3} \neq 0$ (15) is diagonalized via unitary transformation to get physical mass eigenstates 


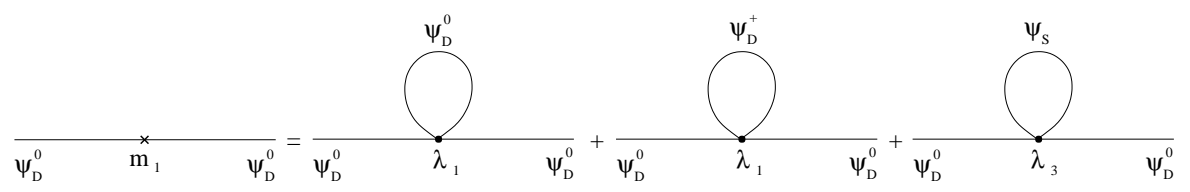

Figure 1. Feynman graphs for the gap equation (17). Similar graphs corresponding to (1618) with exchanged legs and lines.

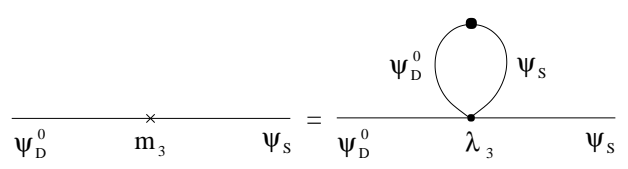

Figure 2. Feynman graphs for the gap equation (19).

$$
\begin{aligned}
& \Psi_{1}=c \Psi_{D}^{0}+s \Psi_{S}, \\
& \Psi_{2}=-s \Psi_{D}^{0}+c \Psi_{S},
\end{aligned}
$$

where $c=\cos \phi$ and $s=\sin \phi, \phi$ is the mixing angle. The masses of the physical fermions $\Psi_{1}, \Psi_{2}$ are

$$
2 M_{1,2}=m_{1}+m_{2} \pm \frac{m_{1}-m_{2}}{\cos 2 \phi} .
$$

The mixing angle is defined by

$$
2 m_{3}=\left(m_{1}-m_{2}\right) \tan 2 \phi .
$$

Again we see, once $m_{3}=0$ the mixing angle vanishes (for $m_{1} \neq m_{2}$ ), $M_{1}=m_{1}$ and $M_{2}=m_{2}$. The physical masses may be equal $\left(M_{1}=M_{2}\right)$ only if $m_{1}=m_{2}$, the original neutral fermions are degenerate in mass and then the mixing angle is meaningless from the point of view of mass matrix diagonalization.

It follows that the physical eigenstates themselves form condensates since

$$
\begin{aligned}
& c^{2}\left\langle\bar{\Psi}_{1 \alpha} \Psi_{1 \beta}\right\rangle_{0}+s^{2}\left\langle\bar{\Psi}_{2 \alpha} \Psi_{2 \beta}\right\rangle_{0}=a_{1} \delta_{\alpha \beta}, \\
& s^{2}\left\langle\bar{\Psi}_{1 \alpha} \Psi_{1 \beta}\right\rangle_{0}+c^{2}\left\langle\bar{\Psi}_{2 \alpha} \Psi_{2 \beta}\right\rangle_{0}=a_{2} \delta_{\alpha \beta}, \\
& c s\left\langle\bar{\Psi}_{1 \alpha} \Psi_{1 \beta}\right\rangle_{0}-c s\left\langle\bar{\Psi}_{2 \alpha} \Psi_{2 \beta}\right\rangle_{0}=a_{3} \delta_{\alpha \beta} .
\end{aligned}
$$

There is no non-diagonal condesate as $\Psi_{1}, \Psi_{2}$ are independent. Combining the equations of (23) one finds

$$
a_{3}=\frac{1}{2} \tan 2 \phi\left(a_{1}-a_{2}\right) .
$$

For $a_{1}=a_{2}, a_{3} \neq 0$ is not possible for $\cos 2 \phi \neq 0$. As is seen, (24) is equivalent to $\left\langle\bar{\Psi}_{1 \alpha} \Psi_{2 \beta}\right\rangle_{0}=0$. Comparing (24) to (22) yields

$$
m_{1}-m_{2}=2 \lambda_{3}\left(a_{1}-a_{2}\right) .
$$


Using the equations (16 19) we are lead to a consistency conditions

$$
\left(\lambda_{3}-\lambda_{1}\right)\left(a_{1}+\frac{4}{3} a_{+}\right)=\left(\lambda_{3}-\lambda_{2}\right) a_{2},
$$

$\lambda_{1} \neq \lambda_{2}$ goes with $a_{1}+\frac{4}{3} a_{+} \neq a_{2}$.

The equations (16 19) can be formulated as gap equations in terms of the physical fields expressing both the masses and the condensates with $\Psi_{1}, \Psi_{2}$ and $\Psi_{+} \equiv \Psi_{D}^{+}$. Assuming vanishing original masses, $m_{0 S}=0, m_{0 D}=0$, the complete set of gap equations are

$$
\begin{aligned}
c \cdot s\left(M_{1}-M_{2}\right) & =2 \lambda_{3} c \cdot s\left(I_{1}-I_{2}\right), \\
c^{2} M_{1}+s^{2} M_{2} & =-\lambda_{1}\left(6\left(c^{2} I_{1}+s^{2} I_{2}\right)+8 I_{+}\right)-8 \lambda_{3}\left(s^{2} I_{1}+c^{2} I_{2}\right), \\
s^{2} M_{1}+c^{2} M_{2} & =-6 \lambda_{2}\left(s^{2} I_{1}+c^{2} I_{2}\right)-8 \lambda_{3}\left(c^{2} I_{1}+s^{2} I_{2}+I_{+}\right), \\
M_{+} & =-\lambda_{1}\left(8\left(c^{2} I_{1}+s^{2} I_{2}\right)+6 I_{+}\right)-8 \lambda_{3}\left(s^{2} I_{1}+c^{2} I_{2}\right) .
\end{aligned}
$$

The main task of the present work is to explore the structure of the gap equations. There are four algebraic equations for four variables $M_{1}, M_{2}, M_{+}$, $c^{2}=\cos ^{2} \phi$. As in almost all approximation $I_{i} \sim M_{i}$, (2730) show gap equation characteristics, $M_{i}=0$ is always a symmetric solution, which is stable for small $\left|\lambda_{i}\right|$. Increasing $\left|\lambda_{i}\right|$ also an energetically favoured [7] massive solution emerges as in the original Nambu Jona-Lasinio model. Now we explore the parameter space $\lambda_{i}$ to find acceptable phyical masses.

Let the condensates be approximated by free field propagators

$$
\left\langle\bar{\Psi}_{i \alpha} \Psi_{i \beta}\right\rangle=\frac{\delta_{\alpha \beta}}{4} I_{i}=-\frac{\delta_{\alpha \beta}}{8 \pi^{2}} M_{i}\left(\Lambda^{2}-M_{i}^{2} \ln \left(1+\frac{\Lambda^{2}}{M_{i}^{2}}\right)\right), \quad i=1,2,+,
$$

where $M_{+}=m_{+}$. Here $\Lambda$ is a four-dimensional physical cutoff, it sets the scale of the new physics responsible for the non-renormalizable operators. From the point ov view of symmetry breaking $\Lambda$ can be chosen arbitrary large (below the GUT or Planck scale), but higher $\Lambda$ implies stronger fine tuning of $\lambda_{3}$, see (32), to keep the new fermion masses in the electroweak range. To avoid fine tuning and allow reasonable fermion masses $\Lambda$ is expected to be a few TeV, typically around $3 \mathrm{TeV}[5]$.

For the electroweak symmetry breaking the most important equation is (27), it triggers mixing between the different representations of the weak gauge group. Applying (31) it reads

$$
0=\left(M_{1}-M_{2}\right) c \cdot s\left(\frac{1}{\lambda_{3}}+\frac{\Lambda^{2}}{\pi^{2}}-\frac{M_{1}^{3} \ln \left(1+\frac{\Lambda^{2}}{M_{1}^{2}}\right)-M_{2}^{3} \ln \left(1+\frac{\Lambda^{2}}{M_{2}^{2}}\right)}{M_{1}-M_{2}}\right) .
$$

(32) always has a symmetric solution $\left(M_{1}-M_{2}\right) c \cdot s=0$, implying $\sin 2 \phi=0$ for $M_{1} \neq M_{2}$, essentialy no mixing, $M_{1}=M_{2}$ is discussed after (22). If $\left|\lambda_{3}\right|$ is greater than a critical value $\left|\lambda_{3}^{c}\right|=\frac{\pi^{2}}{\Lambda^{2}}$ there also exists a symmetry breaking solution $\left(M_{1} \neq M_{2}\right)$, which always has lower energy if the massive solution exists [7]. Equation (32) has a solution with moderate masses $\left(M_{1,2}<0.7 \Lambda\right)$ if $\lambda_{3}$ is negative. In the small mass limit the parantheses in (32) simplifies to 


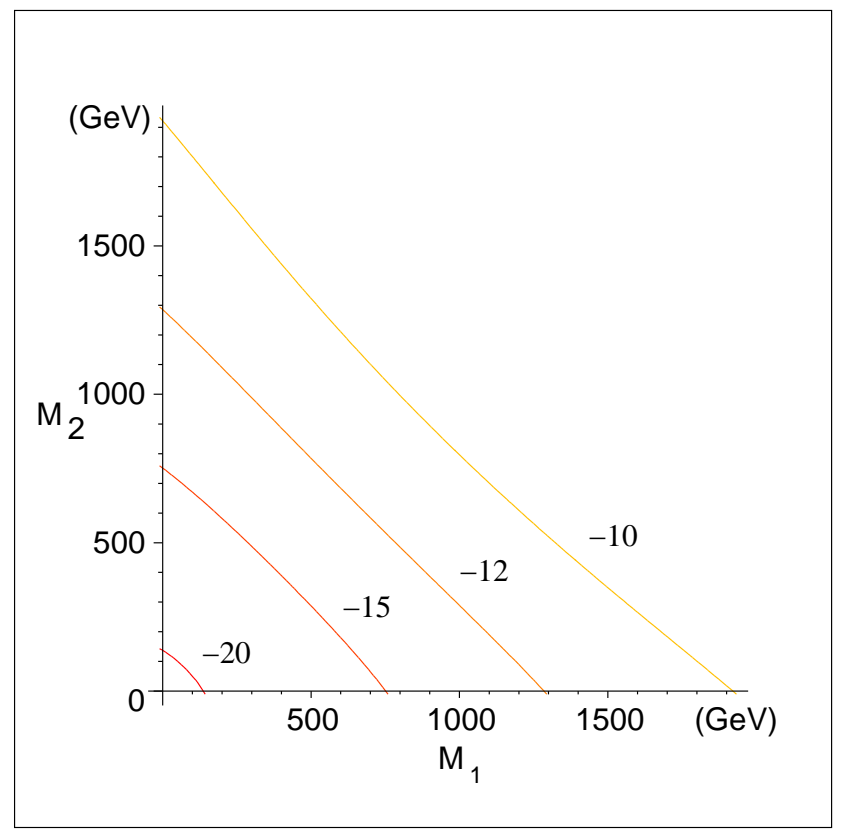

Figure 3. Constant $\lambda_{3}$ contours in the $M_{1}-M_{2}$ plane for $\lambda_{3}=\{-10,-12,-15,-20\} \cdot 1 / \Lambda^{2}, \Lambda=3 \mathrm{TeV}$.

$\frac{1}{\lambda_{3}}+\frac{\Lambda^{2}}{\pi^{2}}-\left(M_{1}^{2}+M_{1} M_{2}+M_{2}^{2}\right)\left(\ln \left(\Lambda^{2}\right)-\ln \left(\tilde{M}^{2}\right)\right)$ where $\tilde{M} \simeq \max \left(M_{1}, M_{2}\right)$. If $\left|\lambda_{3}\right|$ is slightly larger than its critical value, then we generally get small masses compared to $\Lambda, M_{1}^{2}+M_{1} M_{2}+M_{2}^{2} \ll \Lambda^{2}$. The critical coupling agrees with the original Nambu-Jona Lasinio value, only a factor of two coming from the definition in the Lagrangian (11), $\lambda_{3}$ also defined differently in [5. If $\left|\lambda_{3}\right|<$ $\left|\lambda_{3}^{c}\right|$ then the parantheses does not vanish in (32), the condensate $a_{3}$ is not formed and $\left(M_{1}-M_{2}\right) c \cdot s=0$. The physical solution is $c \cdot s=0$, there is no meaningful mixing, $\Psi_{S}, \Psi_{D}$ are the physical mass eigenstates, and the electroweak symmetry is not broken.

Despite the complicated structure of the non-linear equations (2730) we get a relatively simple gap equation for $\lambda_{1}$, similar to (32), from (16) $2 \lambda_{1}\left(a_{1}-a_{+}\right)=$ $m_{1}-m_{+}$. In the physical fields we have

$$
M_{+}-c^{2} M_{1}-s^{2} M_{2}=2 \lambda_{1}\left(I_{+}-c^{2} I_{1}-s^{2} I_{2}\right) .
$$

It includes four unknowns, therefore it cannot be analyzed directly. We get a useful restriction solving (27) and (28) for $\lambda_{1}$ and substituting it to (33), relating $M_{1}, M_{2}, M_{+}$and $c^{2}$ independently of the $\lambda_{i}$ 's. Requiring that $0 \leq c^{2} \leq 1$ we get

$$
M_{1} \leq M_{+} \leq M_{2} .
$$

As a result of the logaritmic terms in $I_{i}, M_{+}$is nonlinear in $c^{2}$, while $m_{1}=$ $c^{2} M_{1}+s^{2} M_{2}$. We remark that though (27) and (33) are very similar, for moderate masses $\lambda_{3}$ is always negative, while $\lambda_{1}$ is positive (also $\lambda_{2}>0$ ). In the $c^{2}=1(0)$ limit $M_{+}=M_{1}\left(M_{2}\right)$ and there are cancelations in (27.30). Turning 
back to the symmetric solution of (32) the relation (34) gives $M_{+}=M_{1}=M_{2}$ and the rest of the gap equations set the common mass equal to zero unless the special relation $6\left(\lambda_{3}-\lambda_{2}\right)=8\left(\lambda_{3}-\lambda_{1}\right)$ holds to provide cancellations.

To find the critical value for $\lambda_{1}$ and $\lambda_{2}$ we considered the limit $M_{+} \rightarrow M_{2}=$ $M$ and $M_{1} \rightarrow 0$ then

$$
\lambda_{1}=\frac{1}{7} \frac{\pi^{2}}{\Lambda^{2}-M^{2} \ln \left(1+\frac{\Lambda^{2}}{M^{2}}\right)}, \quad \lambda_{2}=\frac{4}{3} \frac{\pi^{2}}{\Lambda^{2}-M^{2} \ln \left(1+\frac{\Lambda^{2}}{M^{2}}\right)} .
$$

We get the same NJL type expression if we take the limit $M_{+} \rightarrow M_{1}=M$ and $M_{2} \rightarrow 0$. (35) provides massive solutions if $\lambda_{1} \geq \frac{1}{7} \frac{\pi^{2}}{\Lambda^{2}}$ and $\lambda_{2} \geq \frac{4}{3} \frac{\pi^{2}}{\Lambda^{2}}$. Numerical scans show that these are the minimal, critical values for the couplings and can be approximated in special limits. Numerical solutions are shown in Table 1. for cutoff $\Lambda=3 \mathrm{TeV}$. The role of $M_{1}$ and $M_{2}$ can be exchanged together with $c^{2} \leftrightarrow s^{2}$, therefore we chose $M_{1}<M_{2}$ without the loss of generality. As the cutoff is not too high, $3 \mathrm{TeV}$, there is no serious fine tuning in the $\lambda_{i}$ 's to find relatively small masses.

To understand the signs and roughly the factors in $\lambda_{1,2}^{c}$ consider the limit $M_{1} \simeq M_{2} \simeq M_{+} \simeq M$. If $M \ll \Lambda$ then $\lambda_{3} \simeq \lambda_{3}^{c}=-\frac{\pi^{2}}{\Lambda^{2}}$, though in the exact limit (27) becomes singular. We get from (27.30) the relation $14 \lambda_{1}=6 \lambda_{2}+8 \lambda_{3}$ and a single gap equation ( $I=I_{M}$ in (31) )

$$
M=-\left(14 \lambda_{1}+8 \lambda_{3}\right) I .
$$

Small mass solution requires $\tilde{\lambda}=14 \lambda_{1}+8 \lambda_{3}$ to be close to it's critical value $2 \pi^{2} / \Lambda^{2}$ and provides rough estimates $\lambda_{1} \sim \frac{5}{7} \frac{\pi^{2}}{\Lambda^{2}}$ and also $\lambda_{2} \sim 3 \frac{\pi^{2}}{\Lambda^{2}}$ to generate small masses. Numerical solutions also provide general ( $M_{+}$not close to $M_{1}$ or $M_{2}$ ) small masses for couplings close to these values, see Table 1.

In the strongest small mass limit one neglects the logaritmic terms in the condensates (31), and equations (27.30) reduce to a linear homogeneous system of equations. Two conditions emerge to find a nonzero solution for the physical masses

$$
\begin{aligned}
\left(1+2 \lambda_{1} \frac{\Lambda^{2}}{\pi^{2}}\right)\left(M_{+}-c^{2} M_{1}-s^{2} M_{2}\right) & =0 \\
\left(1-14 \lambda_{1} \frac{\Lambda^{2}}{\pi^{2}}\right)\left(1-6 \lambda_{2} \frac{\Lambda^{2}}{\pi^{2}}\right) & =128\left(\lambda_{3} \frac{\Lambda^{2}}{\pi^{2}}\right)^{2}
\end{aligned}
$$

Remember that $m_{+}=M_{+}$and $m_{1}=c^{2} M_{1}+s^{2} M_{2}, m_{2}=s^{2} M_{1}+c^{2} M_{2}$. In case $\lambda_{1} \frac{\Lambda^{2}}{\pi^{2}}=-\frac{1}{2}$ (38) becomes $\left(1-6 \lambda_{2} \frac{\Lambda^{2}}{\pi^{2}}\right)=16\left(\lambda_{3} \frac{\Lambda^{2}}{\pi^{2}}\right)^{2}$ and there is a mass relation $m_{1}+m_{+}=\left(2 \lambda_{3} \frac{\Lambda^{2}}{\pi^{2}}\right) m_{2}$. If $\lambda_{1} \frac{\Lambda^{2}}{\pi^{2}} \neq-\frac{1}{2}$, we get two relations for the masses, $m_{+}=m_{1}=c^{2} M_{1}+s^{2} M_{2}$ and $\frac{m_{1}}{m_{2}}=\frac{1-6 \lambda_{2} \Lambda^{2} / \pi^{2}}{16 \lambda_{3} \Lambda^{2} / \pi^{2}}=\frac{8 \lambda_{3} \Lambda^{2} / \pi^{2}}{1-14 \lambda_{1} \Lambda^{2} / \pi^{2}}$.

We have calculated and presented briefly in [5] the constraints on the parameters of the model from perturbative unitarity [8, 9]. Consider the amplitudes of two particle $\left(\Psi_{D}^{(+)}, \Psi_{D}^{(0)}\right.$ or $\left.\Psi_{S}\right)$ elastic scattering processes and impose $\left|\Re a_{0}\right| \leq 1 / 2$ for the $J=0$ partial wave amplitudes. The contact graph gives the dominant contribution, neglecting the fermion masses for the $\Psi_{D}^{(+)} \Psi_{D}^{(-)}$scattering gives an upper bound on $\lambda_{1}$ coupling, $\left|\lambda_{1}\right| s \leq 8 \pi$, where $s$ is the maximal center of mass energy $\left(M_{+}^{2} \ll s \leq \Lambda^{2}\right)$. 


\begin{tabular}{|c|c|c|c|c|c|c|c|}
\hline$\lambda_{1}\left(\frac{\pi^{2}}{\Lambda^{2}}\right)$ & 0.546 & 0.740 & 0.496 & 0.380 & 0.502 & 0.468 & 0.419 \\
\hline$\lambda_{2}\left(\frac{\pi^{2}}{\Lambda^{2}}\right)$ & 2.540 & $3.11(!)$ & 2.403 & 2.120 & 2.457 & 2.455 & 2.451 \\
\hline$\lambda_{3}\left(\frac{\pi^{2}}{\Lambda^{2}}\right)$ & -1.031 & -1.041 & -1.042 & -1.070 & -1.083 & -1.178 & -1.330 \\
\hline \hline$M_{1}(\mathrm{GeV})$ & 100 & 148 & 100 & 100 & 150 & 200 & 200 \\
\hline$M_{2}(\mathrm{GeV})$ & 150 & 150 & 200 & 300 & 300 & 500 & 800 \\
\hline$M_{+}(\mathrm{GeV})$ & 149 & 149 & 190 & 290 & 290 & 490 & 790 \\
\hline
\end{tabular}

Table 1: Solutions of the gap equations for the cutoff $\Lambda=3 \mathrm{TeV}, \lambda_{i}$ are given in units of $\frac{\pi^{2}}{\Lambda^{2}}$. In the second column $\lambda_{2}$ violates perturbative unitarity.

We cannot always use the small mass limit, as the solution of the gap equations provide higher $\lambda_{i}$ 's for significantly higher masses. Therefore we have calculated different helicity amplitudes [10] for non-vanishing masses. For $\Psi_{a}(1) \bar{\Psi}_{a}(2) \rightarrow \Psi_{a}(3) \bar{\Psi}_{a}(4),(a=0, s,+), M=\lambda_{i}\left[\left(\bar{v}_{2} u_{1}\right)\left(\bar{u}_{3} v_{4}\right)-\left(\bar{u}_{3} u_{1}\right)\left(\bar{v}_{2} v_{4}\right)\right]$, where $\lambda_{i=1,2,3}$ is the only relevant four-fermion coupling. We consider $\Psi_{S}=$ $s \Psi_{1}+c \Psi_{2}$ scattering as a linear combination in the coupled $\Psi_{1}, \Psi_{2}$ channels to employ only $\lambda_{2}$ (and simiarly $\Psi_{D}^{(0)}$ to constrain $\lambda_{1}$ ). The contributions of the $\gamma, Z$ exchange graphs are negligible $\left(\mathcal{O}\left(g^{2}\right) \ll 8 \pi\right)$ because of the extra propagator. There are three different helicity channels, we give the representative helicity amplitudes, these are maximal for the back to back scattering $\left(\theta_{\text {scattering }}^{\{13\}}=\pi\right)$

$$
\begin{aligned}
& M((+-) \rightarrow(+-))=\lambda_{i}\left(s-4 M_{i}^{2}\right), \\
& M((++) \rightarrow(--))=\lambda_{i} s, \\
& M((+-) \rightarrow(-+))=\lambda_{i} 4 M_{i}^{2} .
\end{aligned}
$$

For other scattering angles $|M|$ is smaller than in (40), for example the maximum for $\theta=0$ is $\lambda_{i} 4 M_{i}^{2}$. The mass dependent unitarity bound agrees with the first estimate

$$
\lambda_{i} s \leq 8 \pi,
$$

where $(\mathrm{i}=1,2,3)$ and $s \leq \Lambda^{2}$ is the center of mass energy. The unitarity constraints are most stringent for $\lambda_{2}$, even the equal small mass limit (36) would set $\lambda_{2} \simeq 3 \pi^{2} / \Lambda^{2}$ which is above the maximum allowed by unitarity $8 \pi / \Lambda^{2} \simeq$ $2.55 \cdot \frac{\pi^{2}}{\Lambda^{2}}$. As an example we show a non-physical nearly equal mass solution in the second column of Table 1., which is not allowed by perturbative unitarity. (42) implies an absolute upper bound on the smaller neutral mass, $M_{1} \leq 230$ $\mathrm{GeV}$ for $\Lambda=3 \mathrm{TeV}$ and generally pushes up the charged mass close to $M_{2}$. The $\lambda_{2}$ contours are drawn in the $M_{2}-M_{1}$ plane for $M_{+}=M_{2}-10 \mathrm{GeV}$ in Figure 4.

In conclusion, we explored and solved the gap equations in an extension of the recently proposed fermion condensate model of electroweak interactions [5]. The original fermions mix via the non-diagonal four-fermion term in the non-renormalizable Lagrangian. The gap equations can be formulated in the physical degrees of freedoms and we have given examples of physical masses for various coupling constants. We determined the critical couplings to define the region of massive solutions, $\lambda_{3}<-\pi^{2} / \Lambda^{2}$ is required to break the electroweak symmetry. With a few $\mathrm{TeV}$ cutoff the couplings do not have to be fine tuned to 


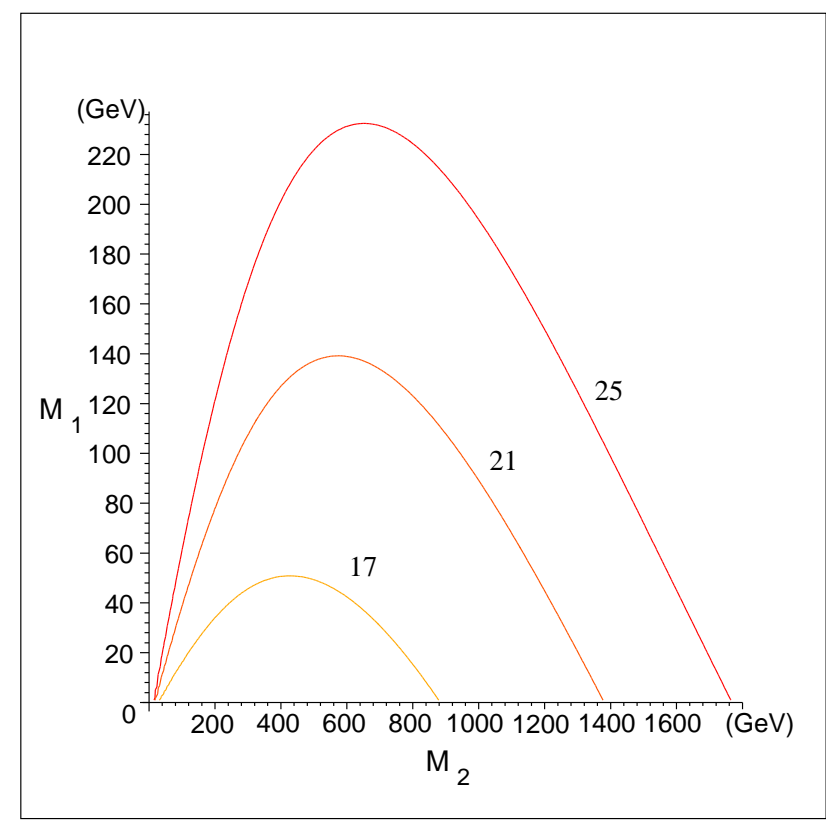

Figure 4. Constant $\lambda_{2}$ contours in the $M_{2}-M_{1}$ plane for

$\lambda_{2}=\{25,21,17\} 1 / \Lambda^{2}$, the allowed region is below the curves, $\Lambda=3 \mathrm{TeV}$.

imply masses of few hundred $\mathrm{GeV}$. In the spectrum three new fermions appear, between the lightest and the heaviest neutral ones there is a charged particle. Perturbative unitarity sets (via the coupling $\lambda_{2}$ ) the mass of the charged fermion relatively close to the heavier neutral one and the lightest fermion mass must be below an upper bound, $M_{1} \leq 230 \mathrm{GeV}$ for $\Lambda=3 \mathrm{TeV}$. The lightest new fermion is stable and a good dark matter candidate as the new fermions interact only in pairs and weakly with standard particles. The model can be tested soon at LHC, numbers of pairs of new particles are expected with masses of few hundred $\mathrm{GeV}$, cross sections for electron-positron colliders are presented in [5].

\section{Acknowledgement}

The authors thank George Pócsik for valuable discussion and collaboration on [5].

\section{References}

[1] Dynamical Symmetry Breaking, World Scientific, 1992, Ed. K. Yamawaki; C.T. Hill and E. H. Simmons, Phys. Rept. 381, 235 (2003); Erratum-ibid. 390, 553 (2004).

[2] W.A. Bardeen, C.T. Hill and M. Lindner, Phys.Rev. D41 1647 (1990); C.T. Hill, Phys.Lett. B266, 419 (1991); M. Lindner and D. Ross, Nucl.Phys. B370, 30 (1992). 
[3] G. Cynolter, E. Lendvai and G. Pócsik, Eur. Phys. J. C38: 247 (2004); Mod.Phys.Lett A9, 1701 (1994).

[4] C. Csáki, C. Grojean, H. Murayama, L.Pilo and J. Terning, Phys. Rev. D 69, 055006 (2004); C. Csáki, C. Grojean, L.Pilo and J. Terning, Phys. Rev. Lett. 92, 101802 (2004); K. Agashe, A. Delgado, M.J. May and R. Sundrum, JHEP 0308, 050 (2003).

[5] G. Cynolter, E. Lendvai and G. Pócsik, Eur. Phys. J. C46: 545 (2006).

[6] Y. Nambu and G. Jona-Lasinio, Phys. Rev. 122, 345 (1961); Y. Nambu and G. Jona-Lasinio, Phys. Rev. 124, 246 (1961).

[7] S. P. Klevansky, Rev. Mod. Phys. 64, No. 3 (1992).

[8] B.Lee, C.Quigg and H.Thacker, Phys. Rev. D16, 1519 (1977); D.Dicus and V.Mathur, Phys. Rev. D7, 3111 (1973).

[9] G. Cynolter, A. Bodor and G. Pócsik, Heavy Ion Phys. 7, 245 (1998).

[10] T. Appelquist, Michael S. Chanowitz, Phys.Rev.Lett. 59, 2405 (1987), Erratum-ibid. 60,1589 (1988). 